\title{
Enhancement Mechanisms of the Second Harmonic Generation from Double Resonant Aluminum Nanostructures
}

\author{
Kuang-Yu Yang, Jérémy Butet, $*$ (1) Chen Yan, Gabriel D. Bernasconi, and Olivier J. F. Martin \\ Nanophotonics and Metrology Laboratory (NAM), Swiss Federal Institute of Technology Lausanne (EPFL), 1015, Lausanne, \\ Switzerland
}

\section{Supporting Information}

\begin{abstract}
Multiresonant plasmonic nanoantennas have recently gained a lot of attention due to their ability to enhance nonlinear optical processes at the nanoscale. The first nanostructure designed for this purpose was an aluminum antenna composed of three arms, designed to be resonant at both the fundamental and the second harmonic frequencies. It was demonstrated that second harmonic generation induced by its resonances at both the fundamental and second harmonic wavelengths is higher than the one from a simple dipolar nanoantenna supporting a resonance at the fundamental wavelength only. However, the underlying mechanisms leading to this strong nonlinear signal are still unclear. In this study, both advanced simulations and experiments are combined to investigate in details the role of the mode coupling in the enhancement of second harmonic generation. By varying the length of the nanoantenna arms, it is clearly demonstrated that second harmonic generation is enhanced when the coupling between the quadrupole and the dipole modes at the second harmonic wavelength is significant. Indeed, using a numerical analysis based on the spatial selection of the second harmonic sources, it is shown that the second harmonic quadrupolar mode, which is directly excited by the fundamental pump, can resonantly transfer its energy to the second harmonic dipolar mode supported by another part of the nanoantenna due to near-field coupling. The study of the second harmonic generation mechanisms of double resonant plasmonic systems is important for the design of efficient second harmonic meta-devices such as coherent extreme-ultraviolet sources, ultrasensitive index, and chiral plasmonic sensors.
\end{abstract}

KEYWORDS: double resonant antenna, nonlinear plasmonics, second harmonic generation, coherent near-ultraviolet source

S econd harmonic generation (SHG) from plasmonic nanostructures has recently gained significant interest thanks to the numerous new possibilities it enables, such as surface-plasmon enhanced nonlinear emission, ${ }^{1-5}$ ultrahigh sensitivity to the nanostructure surface morphology, 6,7 manipulation of the nonlinear radiation through the nanostructure geometry, ${ }^{8,9}$ nonlinear phase control with ultrathin plasmonic metasurfaces, ${ }^{10-12}$ sensing, ${ }^{13-15}$ and abolition of the phase-matching condition. ${ }^{16,17}$ Indeed, plasmonic nanoantennas support localized surface plasmon resonances and can tightly confine light into small volumes. ${ }^{18}$ These induced electromagnetic hot spots, that is, areas where the field is enhanced by several orders of magnitude, can greatly increase the nonlinear signal generated by a nonlinear medium located in nanogaps. ${ }^{19-21}$ Furthermore, the excitation of plasmonic hybridized modes has been found to be a convenient way to control and engineer SHG thanks to the wide spectral tunability of localized surface plasmon resonances. ${ }^{22-24}$ In the case of SHG from plasmonic nanostructures constituted of noble metals, the second harmonic $(\mathrm{SH})$ polarizations mainly originate from the surface of the nanostructures where the inversion symmetry of the crystalline structure is locally broken. $^{25-27}$ Thus, the generated $\mathrm{SH}$ surface polarizations can be used for probing in the far-field morphological defects, down to few nanometers. ${ }^{7}$ The symmetry properties of SHG from coupled plasmonic systems dramatically affect the $\mathrm{SH}$ conversion rate and play an important role since the selection rules differ from that of the excitation field. ${ }^{28}$

So far, most of the previous research works have focused on the SHG with a single engineered plasmonic resonance at either the fundamental or SH wavelength. In both cases, it was clearly shown that the SHG can be enhanced by an order of magnitude in comparison with off-resonance excitation or emission. $^{29-31}$ To further improve the SHG, plasmonic nanostructures supporting resonances at both the fundamental and $\mathrm{SH}$ wavelengths have been recently designed, such as multiresonant or broadband nanostructures. ${ }^{23,24,32-37}$ Interestingly, for the $\mathrm{Al}$ double resonant antennas composed of three$\mathrm{arms}^{32}$ even though the fundamental near-field intensity is lower than that of a dipole nanoantenna resonating only at fundamental wavelength, the $\mathrm{SH}$ scattering intensity is still enhanced several times. ${ }^{32}$ Yet, the physical mechanisms leading to the enhancement of SHG in these nanostructures are still unclear, although essential for the design of efficient nonlinear plasmonic devices.

In this article, we use a surface integral equation (SIE) method $^{38-41}$ and nonlinear optical experiments to study the SHG mechanism in the $\mathrm{Al}$ double resonant antenna composed of three arms (DRA) by detuning the resonance close to the

Received: March 21, 2017

Published: May 8, 2017 
(a)

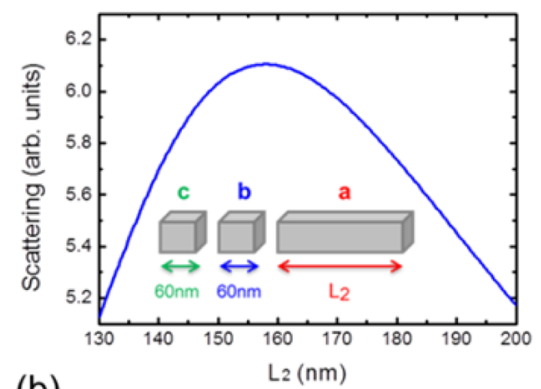

(b)

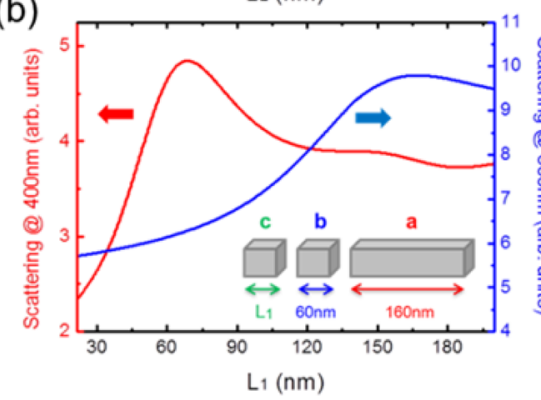

(c)
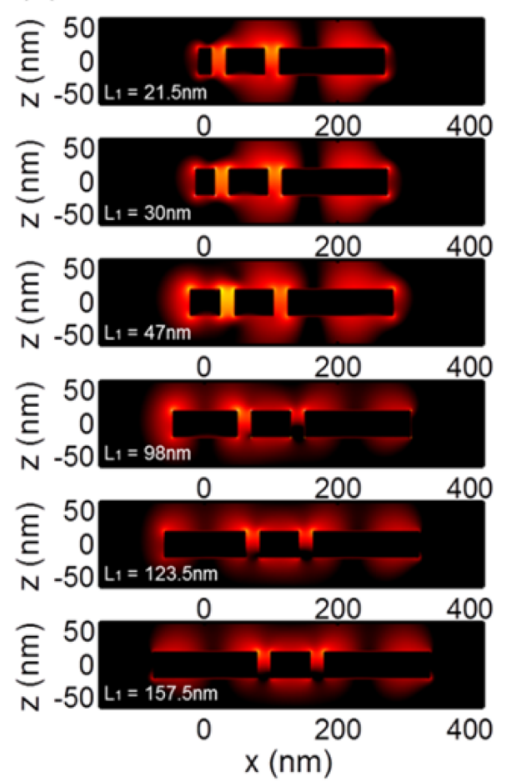

(d)
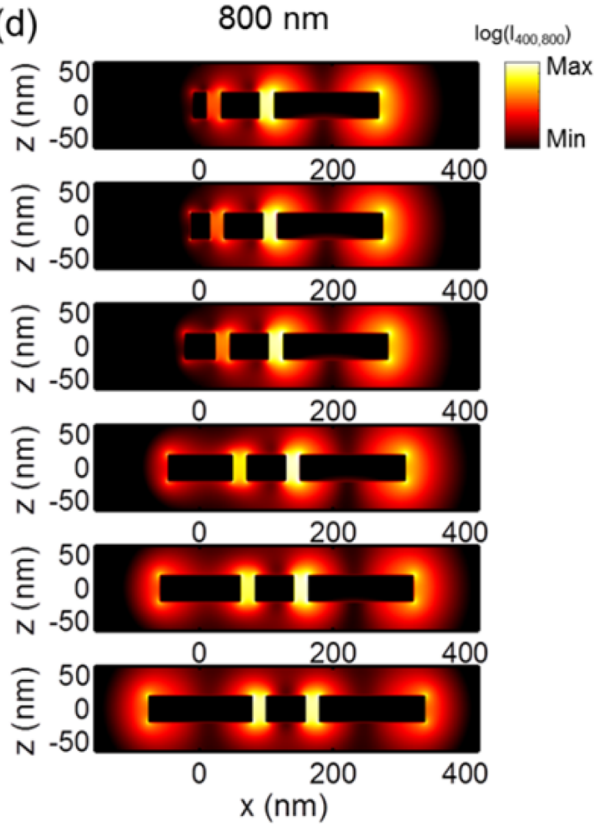

Figure 1. (a) Linear scattering as a function of the length $L_{2}$ of the long nanorod $a$ for an incident wavelength $\lambda=800 \mathrm{~nm}$. The lengths of the middle nanorod $b$ and of the short nanorod $c$ are fixed to $60 \mathrm{~nm}$ here. (b) Linear scattering as a function of the length $L_{1}$ of the short nanorod $c$ for an incident wavelength of 400 (red curve) and $800 \mathrm{~nm}$ (blue curve). Linear near-field intensity (in logarithmic scale) distributions close to the DRAs for six lengths $L_{1}$ considering an incoming planewave with a wavelength (c) $\lambda=400 \mathrm{~nm}$ and (d) $800 \mathrm{~nm}$. From top to bottom: $L_{1}=21.5,30,47,98$, 123.5, and $157.5 \mathrm{~nm}$. For panels (c) and (d), all intensity distributions are plotted with the same colorscale.

$\mathrm{SH}$ wavelength while the fundamental resonance is fixed. The purpose is to evidence the underlying physical mechanisms leading to the enhancement of SHG in DRAs. The DRAs have been fabricated with $\mathrm{Al}$ following a recipe permitting to reduce the defects caused by oxidation, see the Methods section for details. $^{42}$

\section{RESULTS AND DISCUSSION}

Preliminary Design. The DRAs studied in this work consist of three aluminum nanorods aligned along the $x$-axis separated with $20 \mathrm{~nm}$ gaps. The small gap dimension facilitates a strong near-field coupling in both the linear and the nonlinear regimes. Other geometric parameters, namely, the width and thickness of the DRAs, are set to 60 and $40 \mathrm{~nm}$, respectively. The simulations are performed considering isolated structures in a homogeneous background with a refractive index $n=1.45$, corresponding to an averaged value between the refractive indices of water and of the glass substrate. In order to design the DRAs with plasmonic resonances at both fundamental excitation $(800 \mathrm{~nm})$ and the corresponding $\mathrm{SH}$ wavelength (400 nm), the length $L_{2}$ of long rod $a$ is first optimized using SIE simulations. Although the SHG is directly related to the near-field enhancement occurring at the fundamental wavelength (not to the scattering), ${ }^{23}$ here we choose to consider the scattering for the sake of simplicity, since the shift between the maxima of the scattering and the near-field enhancement is negligible for a preliminary design. Figure 1a shows the linear scattered intensity as a function of the length $L_{2}$ for an incoming planewave with a wavelength $\lambda=800 \mathrm{~nm}$. The incident planewave comes at normal incidence and is polarized along the antenna arms. Please note that the lengths of the two short nanorods are here fixed to $60 \mathrm{~nm}$. A maximum of the scattered intensity is observed when the length $L_{2}$ is about 160 $\mathrm{nm}$, corresponding to a strong resonant excitation of a dipolar mode in the long nanorod. In a second step, the length $L_{2}$ is fixed at $160 \mathrm{~nm}$ in order to generate the maximum fundamental near-field intensity close to the DRA and only the length $L_{1}$ of short nanorod $c$ is changed in order to tune a second resonance close to the $\mathrm{SH}$ wavelength $(400 \mathrm{~nm})$. Figure $1 \mathrm{~b}$ shows the linear scattering intensity for different lengths $L_{1}$ ranging from 20 to $200 \mathrm{~nm}$. The red and blue curves represent the variation of the scattering intensity for a planewave excitation at $\lambda=400$ and $800 \mathrm{~nm}$, respectively. The red curve clearly indicates that a length $L_{1}$ close to $60 \mathrm{~nm}$ yields to a scattering maximum for an excitation at $400 \mathrm{~nm}$, whereas a maximum of scattering at 800 $\mathrm{nm}$ is observed for $L_{1}$ close $160 \mathrm{~nm}$. Even though the scattering maximum at $400 \mathrm{~nm}$ is not directly related to the efficiency of the SHG, it still translates to an efficient far-field radiation at this wavelength, as stated by the reciprocity theorem, ${ }^{43}$ and we thus expect to observe an enhanced SH emission for lengths $L_{1}$ close to $60 \mathrm{~nm}$.

In order to determine which part of the DRAs is active under 400 and $800 \mathrm{~nm}$ planewave excitation, the near-field intensity distributions are computed in the $O x z$ plane and are plotted in Figure $1 \mathrm{c}$ and d, respectively, for six lengths $L_{1}$ ranging from 21.5 to $157.5 \mathrm{~nm}$. For an illumination wavelength $\lambda=400 \mathrm{~nm}$, the long nanorod $a$ is weakly excited for this range of lengths $L_{1}$, while a plasmonic dipolar mode with a strong enhancement of the electric field is observed on the short nanorod $c$ when $L_{1}$ $=47 \mathrm{~nm}$. In contrast, considering a $\lambda=800 \mathrm{~nm}$ planewave excitation, a strong dipolar plasmonic mode is excited on the long nanorod $a$, inducing a large electric near-field. Furthermore, the off-resonance behavior of the short nanorod $c$ at the fundamental wavelength is confirmed for the asymmetric cases (short $L_{1}$ ).

Experimental Results. To experimentally investigate the SHG from DRAs, samples were fabricated on a glass substrate with electron beam writer with $100 \mathrm{keV}$ electron gun (Vistec EBPG5000), followed by a lift-off. In order to improve the morphologic uniformity of aluminum nanoantennas, the 
(a)

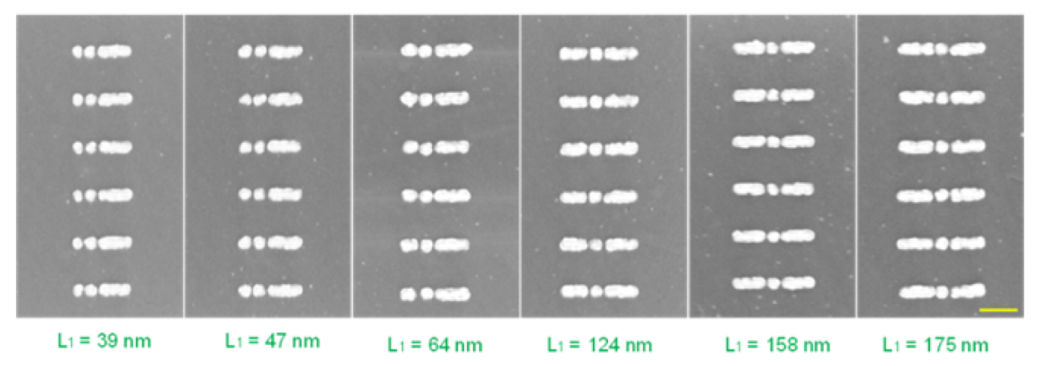

(b)

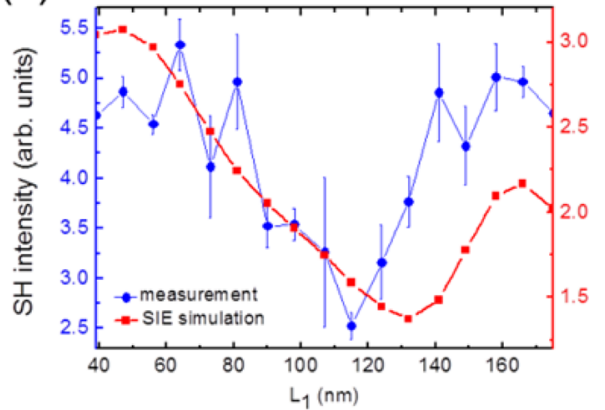

Figure 2. (a) Scanning electron microscopy (SEM) images of aluminum DRAs. Line arrays for six selected lengths $L_{1}$ are shown $\left(L_{1}=39,47,64\right.$, 124,158 , and $175 \mathrm{~nm}$ ). The scale bar at the right bottom corner corresponds to $200 \mathrm{~nm}$. (b) Measured and SIE simulated SH scattering intensity as functions of the length $L_{1}$. The error bars show the maximal and minimal collected SH intensity for each nanoantenna line arrays. Five different positions have been considered for each array. For the SIE simulations, the collection angle is adjusted to fit the experimental detection angle (48. $8^{\circ}$ with the normal).

oxidation of aluminum was carefully prevented during the electron beam lithography (EBL). ${ }^{42}$ Three-arms Al nanoantennas with lengths $L_{1}$ ranging from 39 to $175 \mathrm{~nm}$ have been fabricated. The nanoantenna width and thickness are fixed to 60 and $40 \mathrm{~nm}$, respectively. Figure 2a shows the SEM images of DRAs with different lengths $L_{1}$ revealing high quality nanostructures. The DRAs are arranged in single line arrays and, for each line, the distance between the antennas is $300 \mathrm{~nm}$. For the nonlinear optical measurements, a multiphoton scanning microscopy (LEICA SP5MULTI-PHOTON), combined with a Ti:sapphire femtosecond laser working at $800 \mathrm{~nm}$ with 140 fs pulse duration, is used (see Supporting Information, Figure S1). Figure $\mathrm{S} 1$ also shows the $\mathrm{SH}$ backward intensity image. From each bright line, the $\mathrm{SH}$ signal can be extracted permitting to plot the $\mathrm{SH}$ intensity as a function of the length $L_{1}$. In addition, a quadratic input power dependence of the recorded nonlinear signal is observed for selected arrays $\left(L_{1}=\right.$ 47, 115, and $158 \mathrm{~nm}$ ), confirming that the recorded signal corresponds to SHG (Supporting Information, Figure S2). Note that the two-photon photoluminescence, which is spectrally well-separated from the $\mathrm{SH}$ peak, ${ }^{32,35}$ has been removed with the adequate filters. As the length $L_{1}$ increases, a first maximum is observed between 60 and $80 \mathrm{~nm}$, then the signal intensity decreased by roughly one-half when $L_{1}=115$ $\mathrm{nm}$ and rises again close to the symmetric case $\left(L_{1}=160 \mathrm{~nm}\right)$ due to the strong resonance at the fundamental wavelength.

Full-Wave Computation of the SHG. To support these experimental observations, the SHG from the DRA has been computed using a SIE method (see the Methods section for the implementation details), showing an excellent agreement with the nonlinear measurements (see Figure $2 \mathrm{~b}$ ). The $\mathrm{SH}$ intensity is maximum when $L_{1}=47 \mathrm{~nm}$, almost matching the linear scattering peak at $400 \mathrm{~nm}$ (compare the gray dashed curve in Figure $3 \mathrm{a}$ with Figure $1 \mathrm{~b}$ ). The slight discrepancy between these two values probably comes from the distinct physical natures of SHG and linear scattering. However, it is appealing that the DRAs giving the highest SHG can be roughly estimated with linear far-field excitations, ${ }^{10}$ although the $\mathrm{SH}$ sources originate from their surface. ${ }^{25,26}$ As the length $L_{1}$ increases, the DRA becomes off-resonance at $400 \mathrm{~nm}$ and the SH intensity decreases dramatically. The SH intensity increases again as $L_{1}$ becomes longer than $130 \mathrm{~nm}$, because the coupling with the pump wave starts then increases, as shown in Figure $1 b$, in agreement with the Experimental Results.

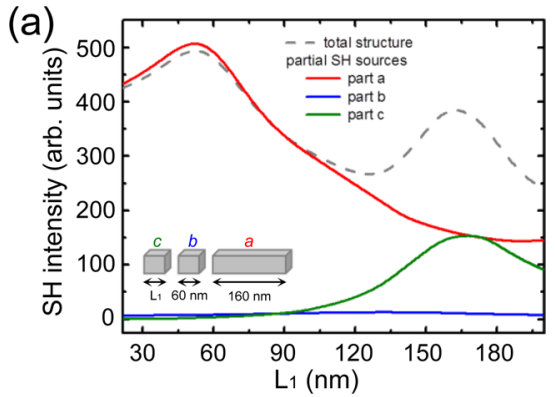

(b)

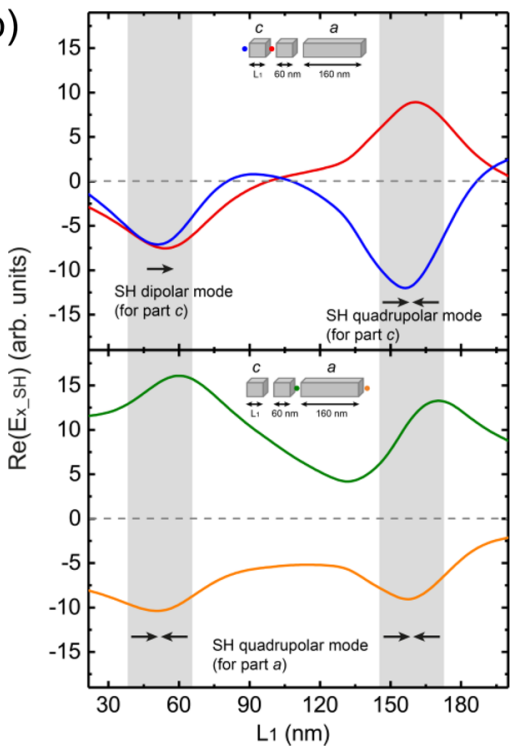

Figure 3. (a) $\mathrm{SH}$ intensity from the total structure (gray dashed curve), long nanorod $a$ (red curve), middle nanorod $b$ (blue curve), and short nanorod $c$ (green curve) as functions of the length $L_{1}$. (b) Real part of the $x$-component of the SH electric fields evaluated at four interesting positions as a function of the length $L_{1}$. The four considered positions are indicated in the inset with two points located at the center of the nanogaps (red and green dots) and two points situated $10 \mathrm{~nm}$ away from both sides of the DRAs (blue and yellow dots). The colored curves indicate the $\mathrm{SH}$ field at each positions a function of the length $L_{1}$. For panel (b), all the $\mathrm{SH}$ sources are considered.

To understand the influence of the length $L_{1}$ on the SHG, the real part of the $x$-component of the $\mathrm{SH}$ electric field $E_{\mathrm{x}} \mathrm{SH}$ was evaluated at four different positions: two at the center of 

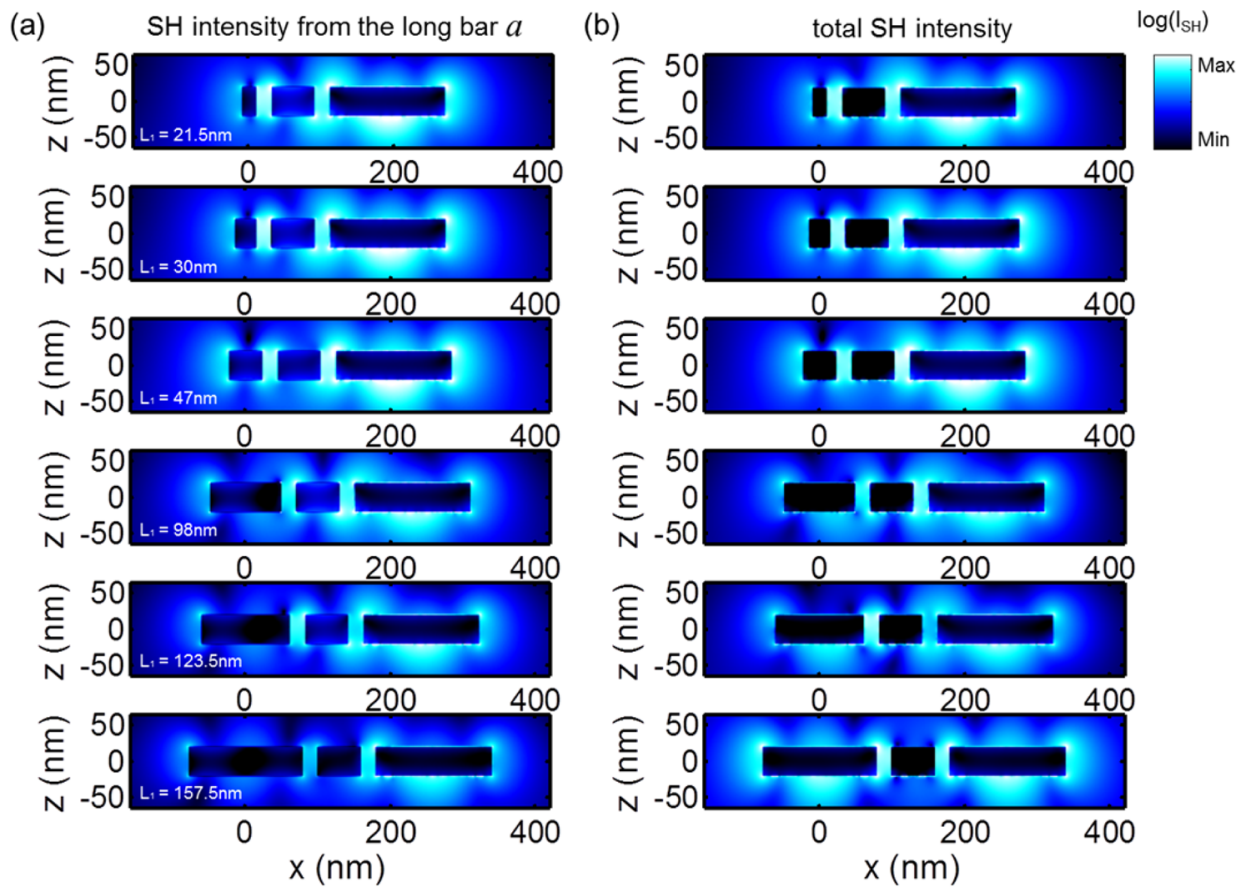

Figure 4. Near-field distributions of the SH intensity for six lengths $L_{1}\left(L_{1}=21.5,30,47,98,123.5\right.$, and 157.5 nm) are considered. (a) Partial SHG from the nanorod $a$ and (b) total SHG from the whole structure. All intensity distributions are plotted with the same colorscale.

the nanogaps and two $10 \mathrm{~nm}$ away from each extremities of the DRA, as shown in the inset of Figure $3 \mathrm{~b}$. The panel on the top presents the variation of $E_{\mathrm{x} \_ \text {SH }}$ close to the short nanorod $c$. The amplitude of the $\mathrm{SH}$ electric fields at these points reach an amplitude maximum around $L_{1}=47 \mathrm{~nm}$. The relative phase $\left(\sim 0^{\circ}\right)$ between the field at those two points indicates that a SH dipolar resonance is supported by the short nanorod $c$, which induces a maximum of the SH scattering as shown in Figure 3a. Two important observations can be made comparing the top and bottom panels of Figure $3 \mathrm{~b}$. First, as $L_{1}$ increases, the $\mathrm{SH}$ mode supported by the short nanorod $c$ gradually evolves from a dipolar nature $\left(L_{1}=47 \mathrm{~nm}\right)$ to a quadrupolar nature (opposite phase between the electric fields evaluated at the extremities), which reaches its maximum in the symmetric case $\left(L_{1}=160 \mathrm{~nm}\right)$ with no real part of the $x$-component of the SH electric field for intermediate lengths (around $L_{1}=98 \mathrm{~nm}$ ). Figure S3 shows the imaginary part of the field at the same points, indicating that the $\mathrm{SH}$ electric field is not vanishing, but is $90^{\circ}$ out of phase relatively to the excitation field. The time evolution of the SH near-field distribution is shown for the symmetric nanoantenna and the DRA as movies (see the Supporting Information). Second, the similar variations of the $\mathrm{SH}$ intensity close to the short nanorod $c$ (top panel) and close to the long nanorod a (bottom panel) indicate a coupling effect. At this point, one can suspect that the SH dipolar mode on the short nanorod $c$ is excited by near-field coupling with the $\mathrm{SH}$ quadrupolar mode on the long nanorod $a$ through the middle nanorod $b$. Indeed, close to $L_{1}=47 \mathrm{~nm}$, the $\mathrm{SH}$ dipolar mode of nanorod $c$ and the quadrupolar mode of nanorod $a$ are maximal, indicating a strong quadrupole-dipole mode coupling. Such a quadrupole-dipole coupling in the SHG from gold nanoantennas has been recently reported by Gennaro et al. ${ }^{37}$ In this work, the authors discussed their experimental data in terms of interferences between dipolar and quadrupolar $\mathrm{SH}$ emissions. Here, we propose to quantitatively address the role of the different constituting elements using a decomposition of the nonlinear sources.

Decomposition of the SH Sources. In order to confirm the importance of the mode coupling for the enhancement of the SHG, a numerical method permitting evaluating the contribution of each constituting part of DRAs was developed (see Methods). This method uses the linearity of the SIE formalism in the undepleted pump approximation and enables analyzing the role of each nanorod in the SHG from coupled plasmonic systems. Note that this self-consistent method considers the near-field coupling between the constituting elements of the DRA at the excitation and emission stages, although the $\mathrm{SH}$ sources stand only on the surface of the considered nanorod, which has a nonzero surface susceptibility $\chi^{(2)}$. In other words, it means that, although one nanorod of the nanostructure does not support $\mathrm{SH}$ sources during the computation, it can still be polarized by the $\mathrm{SH}$ field generated by another part of the nanostructure. The red, blue, and green curves in Figure 3a show the SH far-field scattering intensity as a function of the length $L_{1}$ considering nonlinear sources on the nanorods $a, b$, and $c$, respectively. The comparison between the total $\mathrm{SH}$ intensity (gray dashed curve) with the $\mathrm{SH}$ signal from the long nanorod $a$ (red curve) and the coincidence of the $\mathrm{SH}$ intensity peaks around $L_{1}=47 \mathrm{~nm}$ reveals the mechanism of SHG enhancement in DRA: the near-field SH intensity from the long nanorod $a$ excites the dipolar mode of the short nanorod $c$, increasing the scattering of the $\mathrm{SH}$ intensity into the far-field. In addition, the long nanorod $a$ is found to be the main contribution of the SHG from the DRAs due to its strong resonance at $800 \mathrm{~nm}$. For the nanorod $c$ (green curve), the $\mathrm{SH}$ intensity is gradually increased with the nanorod length $L_{1}$ and reaches a maximum value in the symmetric case, meaning that this part of the nanoantenna is also resonant at the fundamental wavelength in this case. Note that, as expected, the contributions of the nanorods $a$ and $c$ are identical when they have the same length. For the middle nanorod $b$ (blue curve), a 

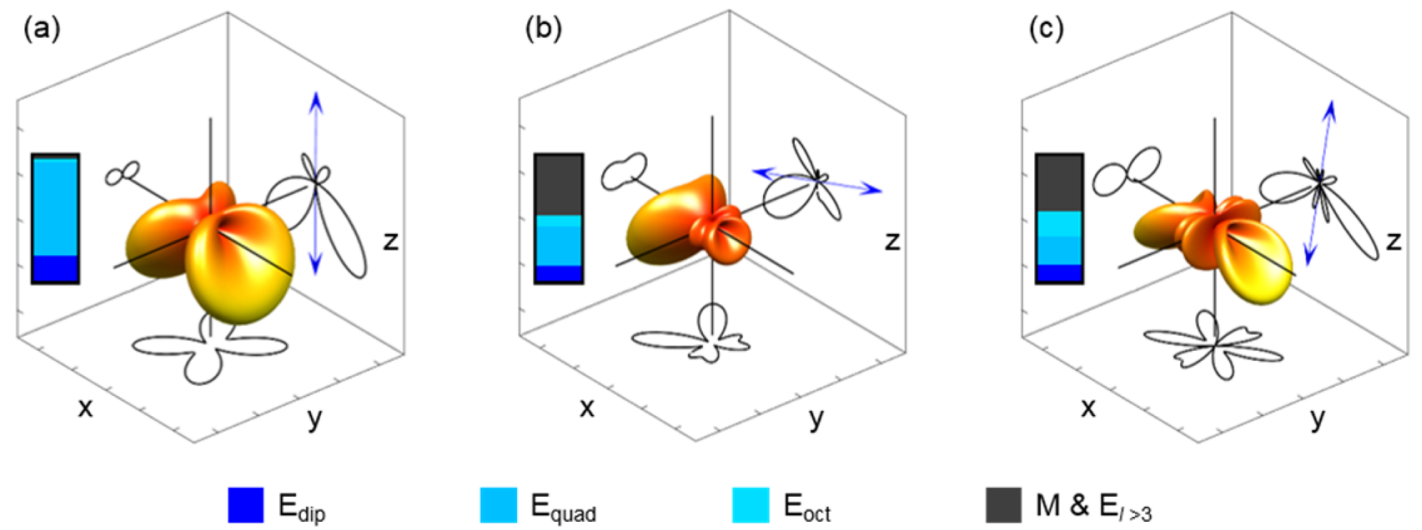

$M \& E_{l>3}$

Figure 5. SH emission patterns and the corresponding multipolar decompositions for (a) a single $\mathrm{Al}$ nanorod $(L=160 \mathrm{~nm}),(\mathrm{b})$ the DRA $\left(L_{1}=47\right.$ $\mathrm{nm})$, and $(\mathrm{c})$ the symmetric antenna $\left(L_{1}=160 \mathrm{~nm}\right)$. The emission patterns have been decomposed into the vector spherical harmonics and the weights of the different orders are shown. The sum of the contributions is normalized to the total SH scattering for each case. $E_{\text {dip }}, E_{\text {quad }}$ and $E_{\text {oct }}$ correspond to the electric dipolar, quadrupolar, and octupolar moments, respectively, whereas $M$ and $E_{k<3}$ correspond to the magnetic moments (always found negligible) and electric moments with higher orders. The blue arrows show the orientation of the dipolar moment. The small $x$ component in the case of the symmetric antenna (c) is due to slight asymmetries existing in the mesh, even though the geometry is symmetric.

(a) $936 \mathrm{~nm}$

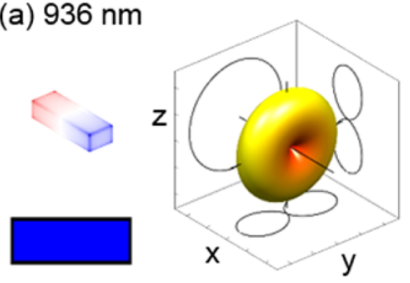

(d) $440 \mathrm{~nm}$

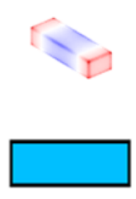

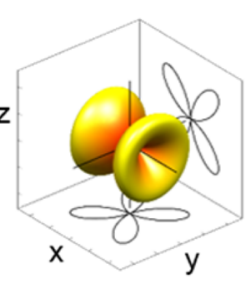

$E_{\text {dip }}$ (b) $909 \mathrm{~nm}$

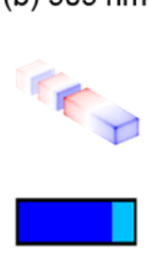

(e) $390 \mathrm{~nm}$

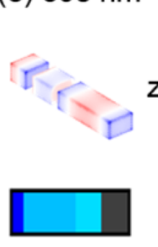

$E_{\text {quad }}$

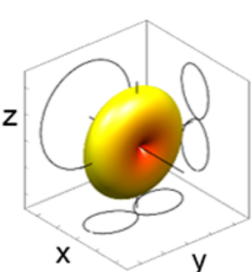

y

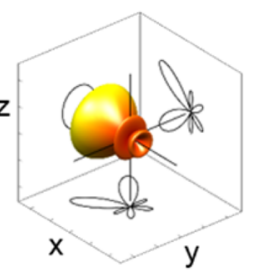

$\mathrm{E}_{\text {oct }}$ (c) $992 \mathrm{~nm}$

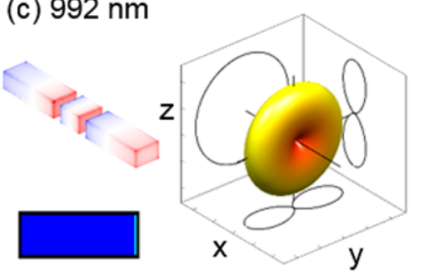

(f) $397 \mathrm{~nm}$

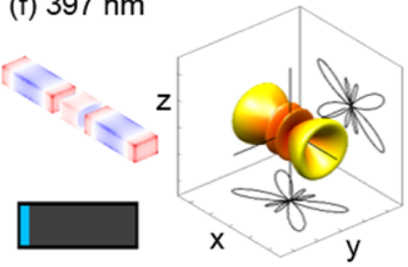

$M \& E_{l>3}$

Figure 6. Fundamental eigenmodes for (a) a single $\mathrm{Al}$ nanorod $(L=160 \mathrm{~nm}),(\mathrm{b})$ the DRA $\left(L_{1}=47 \mathrm{~nm}\right)$, and $(\mathrm{c})$ the symmetric antenna $\left(L_{1}=160\right.$ $\mathrm{nm})$ and modes close to the $\mathrm{SH}$ wavelength $(400 \mathrm{~nm})$ for the three nanostructure. The emission patterns associated with each mode have been decomposed into the vector spherical harmonics and the weights of the different orders are shown as histograms. The sum of the contributions is normalized to the total scattering in each case. $E_{\text {dip, }} E_{\text {quad }}$ and $E_{\text {oct }}$ correspond to the electric dipolar, quadrupolar, and octupolar moments, respectively, whereas $M$ and $E_{l<3}$ corresponds to the magnetic moments (always found negligible) and electric moments with higher orders.

weak $\mathrm{SH}$ intensity is always observed due to an off-resonance behavior at both fundamental and $\mathrm{SH}$ wavelengths.

To further confirm the importance of the mode coupling in the enhancement of the SHG from DRAs, the SH near-field intensity has been evaluated using the SIE method with partial nonlinear sources. The $\mathrm{SH}$ near-field distributions for six lengths $L_{1}$ have been computed considering a nonzero surface susceptibility $\chi^{(2)}$ only for the long nanorod $a$ and for the total antenna, respectively (Figure $4 \mathrm{a}, \mathrm{b}$ ). The $\mathrm{SH}$ near-field distributions with nonzero surface susceptibility $\chi^{(2)}$ for short nanorod $c$ and middle nanorod $b$ are shown in the Supporting Information (Figure S4). These results confirm that the SH sources standing at the surface of the long nanorod are the main contributions to the nonlinear conversion. Indeed, the SH near-field distributions induced by these sources are similar to those observed when all the sources are considered. Furthermore, the $\mathrm{SH}$ near-field distributions resulting from the $\mathrm{SH}$ sources standing at the surface of the other nanorods are much weaker, see Figure S4. From Figure 4a, looking at the case $L_{1}=47 \mathrm{~nm}$, one can see that the resonance of the short bar at $400 \mathrm{~nm}$ leads to a delocalization of the $\mathrm{SH}$ field generated by the long nanorod over the whole DRA. This observation confirms that energy transfer occurs between the $\mathrm{SH}$ sources distributed over the long nanorod surface and the dipolar mode of the short nanorod $c$. Furthermore, we clearly observe that this energy transfer is maximal, that is, the $\mathrm{SH}$ field is maximal close to the short nanorod $c$, when the dipolar mode is tuned close to the $\mathrm{SH}$ wavelength (at $400 \mathrm{~nm}$, corresponding to $L_{1}=47 \mathrm{~nm}$ ).

Multipolar Analysis. In order understand the role of the energy transfer occurring at the $\mathrm{SH}$ wavelength in the enhancement of the SHG from the DRA, a multipolar analysis of the $\mathrm{SH}$ emission has been performed for a single $\mathrm{Al}$ nanorod $(L=160 \mathrm{~nm})$, the asymmetric DRA $\left(L_{1}=47 \mathrm{~nm}\right)$ and the 
symmetric DRA $\left(L_{1}=160 \mathrm{~nm}\right)$. Here, the method used is similar to the one used by Mühlig et al., ${ }^{44}$ except that the SH emission is considered instead of the linear scattering. The case of SHG from a single $\mathrm{Al}$ nanorod is considered first in order to emphasize the specificities of the DRA. The multipolar analysis reveals that the $\mathrm{SH}$ emission corresponds mainly to a quadrupolar emission with a small dipolar contribution, Figure 5a. Note that the dipole moment is aligned along the propagation direction and is due to the retardation effect at the fundamental wavelength, as intensively discussed in the past. $^{29,30,45,46}$ These observations are then prevalent in the SHG from a centrosymmetric object with a small size compared to the wavelength. ${ }^{29,30,45,46}$ Different from a single nanorod, the SHG from the asymmetric and symmetric DRAs involve high order modes. However, two main differences are observed. First, the SHG from the symmetric antenna has larger contributions from the octupolar moment. Second, the orientation of the $\mathrm{SH}$ dipolar moment is not the same for both structures. For the symmetric DRA, the $\mathrm{SH}$ dipolar moment is along the propagation direction of the incident wave, as is also the case for the single nanorod, meaning that the excitation of this mode comes from the retardation effects at the fundamental wavelength. This is indeed the only possibility allowed by the symmetry of the problem at hand. On the contrary, the SH dipolar moment supported by the DRA is mainly along the antenna arms (only a small component is induced by the field variation at the pump wavelength). The new orientation of the $\mathrm{SH}$ dipolar moment is a direct consequence of the energy transfer occurring at the $\mathrm{SH}$ wavelength, which is allowed by the symmetry breaking along the $x$-axis. Indeed, as discussed in the previous section, the nonlinear sources standing at the surface of the long nanorod resonantly drive the dipolar mode of the short nanorod at the $\mathrm{SH}$ wavelength, resulting into a dipolar $\mathrm{SH}$ far-field radiation polarized along the antenna axis. Although the DRA gives the highest SHG, it should be mentioned that the ratio between the total SH intensity scattered by the asymmetric DRA and that scattered by the single nanorods or the symmetric DRA are 1.88 or 1.26 . To understand why the enhancement of the SHG is not higher, it was found useful to perform an eigenmode analysis.

Eigenmode Analysis. Using a numerical method for finding the eigenmodes of plasmonic nanostructures based on $\mathrm{SIE}^{36,47}$ the fundamental dipolar mode has been determined for a single $\mathrm{Al}$ nanorod $(L=160 \mathrm{~nm})$, the asymmetric DRA, and the symmetric DRA (Figure 6). For all these nanostructures, the fundamental dipolar mode is observed in the nearinfrared, around $\lambda=950 \mathrm{~nm}$. Note that this mode does not exactly match the pump wavelength, since the preliminary design has been based on the scattering cross-section, with a substantial contribution from other eigenmodes. At the same time, the eigenmode analysis reveals some modes close to the $\mathrm{SH}$ wavelength $(\lambda=400 \mathrm{~nm})$ for all the considered nanostructures. For example, in the case of the single nanorod, a quadrupolar mode is observed at $\lambda=440 \mathrm{~nm}$. This double resonant condition in single $\mathrm{Al}$ nanorod has already been experimentally observed by the Bachelier's group, ${ }^{35}$ following our theoretical discussion on the efficiency of this double resonant condition. ${ }^{36}$ In the case of the symmetric DRA, a mode is observed at $\lambda=397 \mathrm{~nm}$. This mode is composed of quadrupolar modes supported by each long nanorod. It is interesting to note that this mode has the appropriate parity for contributing to the $\mathrm{SH}$ emission. On the other hand, considering the modes close the SH frequency, the asymmetric DRA is the only one that exhibits a net dipolar contribution. Indeed, since there is no excitation field, the two fully centrosymmetric nanostructures, that is, the single nanorod and symmetric nanoantenna, cannot exhibit dipolar contributions for those even order modes. This eigenmode analysis reveals that, to some extent, all the nanostructures considered here satisfy a double resonant condition. This is a direct consequence of the use of $\mathrm{Al}$ as the plasmonic material: Indeed, $\mathrm{Al}$ is the material of choice for plasmonic modes in the near-UV and the observation of localized surface plasmon resonances close to $\lambda=400 \mathrm{~nm}$ is common in nanostructures made of $\mathrm{Al}$ with various shapes, ${ }^{48-54}$ meaning that the double resonant condition is easily achieved with this material for a pump at $\lambda=$ $800 \mathrm{~nm}$. Since the comparison is performed between double resonant antennas, a SH enhancement of 1.5 for the DRA is already meaningful, pointing out the importance of the energy transfer at the $\mathrm{SH}$ wavelength in the $\mathrm{SH}$ enhancement.

\section{CONCLUSION}

In summary, a comprehensive study of the mechanisms leading to the enhancement of SHG in double-resonant plasmonic nanoantennas has been performed. By combining full wave SIE simulations with advanced fabrication techniques and sensitive nonlinear optical experiments on $\mathrm{Al}$ plasmonic nanostructures, the modulation of SHG from three-arm aluminum nanoantennas has been studied for a broad variety of geometries. For this purpose, the length of the long nanorod $a$ of the DRAs has been first optimized and fixed at $160 \mathrm{~nm}$, while the length of the nanorod at other extremity is varied from 20 to $200 \mathrm{~nm}$. It has been clearly observed that the $\mathrm{SH}$ intensity is maximum when this nanorod is able to scatter light at the $\mathrm{SH}$ wavelength. The $\mathrm{SH}$ intensity observed in this case is almost twice the value obtained when the short nanorod $c$ does not have any resonant behavior at $400 \mathrm{~nm}$. The corresponding $\mathrm{SH}$ near-field distributions confirm the presence of a quadrupolar $\mathrm{SH}$ supported by the long nanorod, which is able to drive a dipolar resonance supported by another nanoantenna arm. This energy transfer during the frequency conversion process is summarized in Figure 7 (the case of the symmetric nanoantenna is shown in Figure S5) and represents a new way for increasing and controlling nonlinear optical conversion at the nanoscale. Indeed, contrary to other designs, ${ }^{37}$ the interplay between the dipolar and quadrupolar moments is found beneficial for the SHG. These results provide new physical insights into the physical mechanisms of the SHG enhancement in multiresonant plasmonic structures and should guide their utilization for the design of efficient nonlinear plasmonic metasurfaces based on double resonant nanostructures.

\section{METHODS}

Simulation. The linear and SHG simulations are performed with an in-house code based on the SIE formulation. The $\mathrm{Al}$ permittivity has been extrapolated from experimental data. ${ }^{50}$ For the linear SIE evaluation, the electric and magnetic surface currents are expanded on the Rao-Wilton-Glisson (RWG) basis functions with discretized triangular meshes approximating the boundary surface. The expanding coefficients are obtained by enforcing the boundary conditions on the surfaces and the Galerkin's method. A Poggio-Miller-Chang-Harrington-Wu-Tsai method is applied to ensure the accuracy of the solutions. The electric and magnetic fields are then deduced 


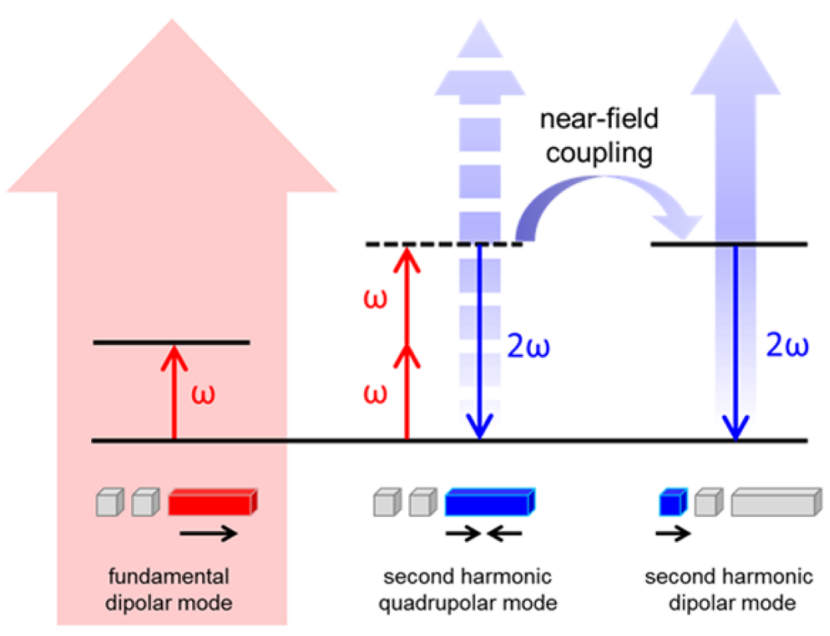

Figure 7. Mechanisms of the SHG enhancement in DRA with both fundamental (long nanorod) and SH (short nanorod) resonances. The fundamental excitation, the direct $\mathrm{SH}$ quadrupolar emission (long nanorod) and SH dipolar mode (short nanorod) are depicted as a red arrow, a blue dashed arrow, and a blue arrow, respectively. Note that the near-field coupling occurs when the short nanorod supports a dipolar mode at the SH wavelength.

from the evaluation of the Green's functions. The surface SH polarization can be written as

$$
P\left(r^{+}\right)=\chi_{\perp \perp \perp}^{(2)}: E\left(r^{-}\right) E\left(r^{-}\right)
$$

Here we only consider the component of surface tensor normal

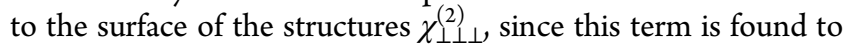
dominate the nonlinear response of metallic nanoparticles. For the computation of the $\mathrm{SH}$ polarization, the linear electric fields is evaluated just below the aluminum surface. The $r^{+}$and $r^{-}$ denote the locations just above and below the interface, respectively. Once the $\mathrm{SH}$ polarization is calculated, the $\mathrm{SH}$ surface currents are yielded by solving the SIE formulation taking into account boundary conditions including the nonlinear polarization. The $\mathrm{SH}$ electric field $E^{\mathrm{SH}}(r)$ and magnetic field $\mathrm{H}^{\mathrm{SH}}(r)$ at any point of space can then be computed,

$$
\begin{aligned}
& E^{\mathrm{SH}}(r)=-i 2 \omega \mu \int \mathrm{d} S^{\prime} G^{\mathrm{SH}}\left(r^{\prime}, r\right) \cdot J^{\mathrm{SH}}\left(r^{\prime}\right) \\
& -\int \mathrm{d} S^{\prime}\left[\nabla^{\prime} \times G^{\mathrm{SH}}\left(r^{\prime}, r\right)\right] \cdot M^{\mathrm{SH}}\left(r^{\prime}\right) \\
& H^{\mathrm{SH}}(r)=-i 2 \omega \varepsilon \int \mathrm{d} S^{\prime} G^{\mathrm{SH}}\left(r^{\prime}, r\right) \cdot M^{\mathrm{SH}}\left(r^{\prime}\right) \\
& \quad+\int \mathrm{d} S^{\prime}\left[\nabla^{\prime} \times G^{\mathrm{SH}}\left(r^{\prime}, r\right)\right] \cdot J^{\mathrm{SH}}\left(r^{\prime}\right)
\end{aligned}
$$

where $J^{\mathrm{SH}}$ and $M^{\mathrm{SH}}$ represent the electric and magnetic surface current densities, respectively, and $\mathrm{d} S^{\prime}$ stands for the boundary surfaces of all embedded domains. Furthermore, a numerical method permitting to consider a nonzero surface polarization $\chi_{\perp \perp}^{(2)}$ on specific parts of the DRAs has been developed in order to facilitate the analysis of SHG from strongly coupled systems. In this case, the nonlinear surface currents are driven on the total structure by $\mathrm{SH}$ sources located at the surface of the selected parts only.

Fabrication. For the fabrication of aluminum nanostructures, it was observed that residual gases, especially $\mathrm{H}_{2} \mathrm{O}$ and $\mathrm{O}_{2}$ have a strong dissociative absorption leading to the formation of aluminum oxide, which deteriorates the morphology of the nanostructures. Therefore, $\mathrm{H}_{2} \mathrm{O}$ present in the glass substrate, the spin-coated photoresist, and the evaporation environment should be minimized as much as possible. Thus, an optimized recipe for aluminum nanostructures was developed. Thus, an optimized recipe for the fabrication of aluminum nanostructures was developed as detailed in ref 42 and utilized for the fabrication of all the nanostructures in this study.

Nonlinear Optical Measurement. For the nonlinear optical measurements, we use a multiphoton scanning microscopy (LEICA SP5MULTI-PHOTON) combined with a 20x/1.00 NA water-immersion objective (HCX PL APO) and a Ti:sapphire femtosecond laser (Chameleon ultra laser with a $80 \mathrm{MHz}$ repetition rate) operating at $\lambda=800 \mathrm{~nm}$ with a $140 \mathrm{fs}$ pulse duration combined with an electro-optical modulator (EOM) to adjust the input power. The backward scattered SHG from the structures is collected by the same objective followed by a beam splitter, a band-pass filter (400/15 BrightLine fluorescence filter), and a photomultiplier tube (NDD PMT). The XY scanner (400 Hz scanning speed) of the microscopy enables a $387.5 \mu \mathrm{m} \times 387.5 \mu \mathrm{m}$ field range with $758.32 \mathrm{~nm}$ pixel resolution. The quadratic dependence between the $\mathrm{SH}$ intensity and the input power has been recorded by carefully tuning the input power.

\section{ASSOCIATED CONTENT}

\section{Supporting Information}

The Supporting Information is available free of charge on the ACS Publications website at DOI: 10.1021/acsphotonics.7b00288.

Schematic of the experimental setup, power dependence of the collected signal, near-field distributions of the second harmonic intensity, schematic of the SHG from the symmetric DRA (PDF).

Movie showing the time evolution of the second harmonic intensity for the asymmetric DRA (AVI).

Movie showing the time evolution of the second harmonic intensity for the symmetric DRA (AVI).

\section{AUTHOR INFORMATION}

\section{Corresponding Author}

*E-mail: jeremy.butet@epfl.ch.

ORCID

Jérémy Butet: 0000-0001-9598-9074

\section{Author Contributions}

K.-Y.Y. ran the numerical simulations, fabricated the samples, and performed the nonlinear optical measurements. J.B. developed the SIE method for SHG, provided assistance in the numerical simulations and the nonlinear optical measurements, and wrote the manuscript. G.D.B. performed both the eigenmode and multipole analysis. C.Y. wrote the code for the multipolar analysis. O.J.F.M. developed the concept of DRA for increasing the SHG in plasmonic systems and supervised the project. All the authors discussed the results and contributed to the final version of the manuscript.

\section{Notes}

The authors declare no competing financial interest.

\section{ACKNOWLEDGMENTS}

It is a pleasure to acknowledge T. Laroche for his help with the nonlinear optical measurements as well as funding from the Swiss National Science Foundation (Projects 200020_153662 
and 200021L 156184) and from the European Research Council (ERC-2015-AdG-695206 Nanofactory)

\section{REFERENCES}

(1) Butet, J.; Brevet, P.-F.; Martin, O. J. F. Optical Second Harmonic Generation in Plasmonic Nanostructures: From Fundamental Principles to Advanced Applications. ACS Nano 2015, 9, 1054510562.

(2) Czaplicki, R.; Husu, H.; Siikanen, R.; Mäkitalo, J.; Kauranen, M. Enhancement of second-harmonic generation from metal nanoparticles by passive elements. Phys. Rev. Lett. 2013, 110, 093902.

(3) Zhang, Y.; Grady, N. K.; Ayala-Orozco, C.; Halas, N. J. ThreeDimensional Nanostructures as Highly Efficient Generators of Second Harmonic Light. Nano Lett. 2011, 11, 5519-5523.

(4) Butet, J.; Duboisset, J.; Bachelier, G.; Russier-Antoine, I.; Benichou, E.; Jonin, C.; Brevet, P.-F. Optical Second Harmonic Generation of Single Metallic Nanoparticles Embedded in a Homogeneous Medium. Nano Lett. 2010, 10, 1717-1721.

(5) Zhang, S.; Li, G.-C.; Chen, Y.; Zhu, X.; Liu, S.-D.; Lei, D. Y.; Duan, H. Pronounced Fano Resonance in Single Gold Split Nanodisks with $15 \mathrm{~nm}$ Split Gaps for Intensive Second Harmonic Generation. ACS Nano 2016, 10, 11105-11114.

(6) Bautista, G.; Huttunen, M. J.; Mäkitalo, J.; Kontio, J. M.; Simonen, J.; Kauranen, M. Second-Harmonic Generation Imaging of Metal Nano-Objects with Cylindrical Vector Beams. Nano Lett. 2012, $12,3207-3212$.

(7) Butet, J.; Thyagarajan, K.; Martin, O. J. F. Ultrasensitive Optical Shape Characterization of Gold Nanoantennas Using Second Harmonic Generation. Nano Lett. 2013, 13, 1787-1792.

(8) Butet, J.; Martin, O. J. F. Nonlinear Plasmonic Nanorulers. ACS Nano 2014, 8, 4931-4939.

(9) Smirnova, D.; Kivshar, Y. S. Multipolar Nonlinear Nanophotonics. Optica 2016, 3, 1241-1255.

(10) O’Brien, K.; Suchowski, H.; Rho, J.; Salandrino, A.; Boubacar, K.; Yin, X.; Zhang, X. Predicting Nonlinear Properties of Metamaterials from the Linear Response. Nat. Mater. 2015, 14, 379-383.

(11) Segal, N.; Keren-Zur, S.; Hendler, N.; Ellenbogen, T. Controlling Light with Metamaterials-Based Nonlinear Photonic Crystals. Nat. Photonics 2015, 9, 180-184.

(12) Li, G.; Chen, S.; Pholchai, N.; Reineke, B.; Wong, P. W. H.; Pun, E. Y. B.; Cheah, K. W.; Zentgraf, T.; Zhang, S. Continuous Control of the Nonlinearity Phase for Harmonic Generations. Nat. Mater. 2015, 14, 607-612.

(13) Singh, A. K.; Senapati, D.; Wang, S.; Griffin, J.; Neely, A.; Candice, P.; Naylor, K. M.; Varisli, B.; Kalluri, J. R.; Ray, P. C. Gold Nanorod Based Selective Identification of Escherichia coli Bacteria Using Two-Photon Rayleigh Scattering Spectroscopy. ACS Nano 2009, 3, 1906-1912.

(14) Neely, A.; Perry, C.; Varisli, B.; Singh, A.; Arbneshi, T.; Senapati, D.; Kalluri, J. R; Ray, P. C. Ultrasensitive and Highly Selective Detection of Alzheimer's Disease Biomarker Using Two-Photon Rayleigh Scattering Properties of Gold Nanoparticle. ACS Nano 2009, 3, 2834-2840.

(15) Butet, J.; Russier-Antoine, I.; Jonin, C.; Lascoux, N.; Benichou, E.; Brevet, P.-F. Sensing with Multipolar Second Harmonic Generation from Spherical Metallic Nanoparticles. Nano Lett. 2012, 12, 1697-1701.

(16) Rose, A.; Smith, D. R. Overcoming Phase Mismatch in Nonlinear Metamaterials [Invited]. Opt. Mater. Express 2011, 1, 12321243.

(17) Suchowski, H.; O’Brien, K.; Wong, Z. J.; Salandrino, A.; Yin, X.; Zhang, X. Phase Mismatch-Free Nonlinear Propagation in Optical Zero-Index Materials. Science 2013, 342, 1223-1226.

(18) Mühlschlegel, P.; Eisler, H.-J.; Martin, O. J. F.; Hecht, B.; Pohl, D. W. Resonant Optical Antennas. Science 2005, 308, 1607-1609.

(19) Grinblat, G.; Rahmani, M.; Cortés, E.; Caldarola, M.; Comedi, D.; Maier, S. A.; Bragas, A. V. High-Efficiency Second Harmonic
Generation from a Single Hybrid $\mathrm{ZnO}$ Nanowire/Au Plasmonic Nano-Oligomer. Nano Lett. 2014, 14 (2014), 6660-6665.

(20) Aouani, H.; Rahmani, M.; Navarro-Cia, M.; Maier, S. A. ThirdHarmonic-Upconversion Enhancement from a Single Semiconductor Nanoparticle Coupled to a Plasmonic Antenna. Nat. Nanotechnol. 2014, 9, 290-294.

(21) Metzger, B.; Hentschel, M.; Schumacher, T.; Lippitz, M.; Ye, X.; Murray, C. B.; Knabe, B.; Buse, K.; Giessen, H. Doubling the Efficiency of Third Harmonic Generation by Positioning ITO Nanocrystals into the Hot-Spot of Plasmonic Gap-Antennas. Nano Lett. 2014, 14, 2867-2872.

(22) de Ceglia, D.; Vincenti, M. A.; de Angelis, C.; Locatelli, A.; Haus, J. W.; Scalora, M. Role of Antenna Modes and Field Enhancement in Second Harmonic Generation from Dipole Nanoantenna. Opt. Express 2015, 23, 1715-1729.

(23) Thyagarajan, K.; Butet, J.; Martin, O. J. F. Augmenting Second Harmonic Generation Using Fano Resonances in Plasmonic Systems. Nano Lett. 2013, 13, 1847-1851.

(24) Liu, S.-D.; Leong, E. S. P.; Li, G.-C.; Deng, Y. H.; Teng, J. H.; Ong, K. C.; Lei, D. Y. Polarization-Independent Multiple Fano Resonances in Plasmonic Nonamers for Multimode-Matching Enhanced Multiband Second-Harmonic Generation. ACS Nano 2016, 10, 1442-1453.

(25) Wang, F. X.; Rodriguez, F. J.; Albers, W. M.; Ahorinta, R.; Sipe, J. E.; Kauranen, M. Surface and Bulk Contributions to the SecondOrder Nonlinear Optical Response of a Gold Film. Phys. Rev. B: Condens. Matter Mater. Phys. 2009, 80, 233402.

(26) Bachelier, G.; Butet, J.; Russier-Antoine, I.; Jonin, C.; Benichou, E.; Brevet, P.-F. Origin of Optical Second-Harmonic Generation in Spherical Gold Nanoparticles: Local Surface and Nonlocal Bulk Contributions. Phys. Rev. B: Condens. Matter Mater. Phys. 2010, 82, 235403.

(27) Ciracì, C.; Poutrina, E.; Scalora, M.; Smith, D. R. SecondHarmonic Generation in Metallic Nanoparticles: Clarification of the Role of the Surface. Phys. Rev. B: Condens. Matter Mater. Phys. 2012, 86,115451 .

(28) Butet, J.; Dutta-Gupta, S.; Martin, O. J. F. Surface SecondHarmonic Generation from Coupled Spherical Plasmonic Nanoparticles: Eigenmode Analysis and Symmetry Properties. Phys. Rev. B: Condens. Matter Mater. Phys. 2014, 89, 245449.

(29) Dadap, J. I.; Shan, J.; Eisenthal, K. B.; Heinz, T. F. SecondHarmonic Rayleigh Scattering from a Sphere of Centrosymmetric Material. Phys. Rev. Lett. 1999, 83, 4045-4048.

(30) Dadap, J. I.; Shan, J.; Heinz, T. F. Theory of Optical SecondHarmonic Generation from a Sphere of Centrosymmetric Material: Small-Particle Limit. J. Opt. Soc. Am. B 2004, 21, 1328.

(31) Metzger, B.; Gui, L.; Fuchs, J.; Floess, D.; Hentschel, M.; Giessen, H. Strong Enhancement of Second Harmonic Emission by Plasmonic Resonances at the Second Harmonic Wavelength. Nano Lett. 2015, 15, 3917-3922.

(32) Thyagarajan, K.; Rivier, S.; Lovera, A.; Martin, O. J. F. Enhanced Second-Harmonic Generation from Double Resonant Plasmonic Antennae. Opt. Express 2012, 20, 12860-12865.

(33) Aouani, H.; Navarro-Cia, M.; Rahmani, M.; Sidiropoulos, T. P. H.; Hong, M.; Oulton, R. F.; Maier, S. A. Multiresonant Broadband Optical Antennas As Efficient Tunable Nanosources of Second Harmonic Light. Nano Lett. 2012, 12, 4997-5002.

(34) Celebrano, M.; Wu, X.; Baselli, M.; Gro $\beta$ mann, S.; Biagioni, P.; Locatelli, A.; De Angelis, C.; Cerullo, G.; Osellame, R.; Hecht, B.; et al. Mode Matching in Multiresonant Plasmonic Nanoantennas for Enhanced Second Harmonic Generation. Nat. Nanotechnol. 2015, 10, 412-417.

(35) Ethis de Corny, M.; Chauvet, N.; Laurent, G.; Jeannin, M.; Olgeirsson, L.; Drezet, A.; Huant, S.; Dantelle, G.; Nogues, G.; Bachelier, G. Wave-Mixing Origin and Optimization in Single and Compact Aluminum Nanoantennas. ACS Photonics 2016, 3, 18401846. 
(36) Bernasconi, G. D.; Butet, J.; Martin, O. J. F. Mode Analysis of Second-Harmonic Generation in Plasmonic Nanostructures. J. Opt. Soc. Am. B 2016, 33, 768-779.

(37) Gennaro, S. D.; Rahmani, M.; Giannini, V.; Aouani, H.; Sidiropoulos, T. P. H.; Navarro-Cía, M.; Maier, S. A.; Oulton, R. F. The Interplay of Symmetry and Scattering Phase in Second Harmonic Generation from Gold Nanoantennas. Nano Lett. 2016, 16, 52785285.

(38) Kern, A. M.; Martin, O. J. F. Surface Integral Formulation for 3D Simulations of Plasmonic and High Permittivity Nanostructures. J. Opt. Soc. Am. A 2009, 26, 732-740.

(39) Mäkitalo, J.; Suuriniemi, S.; Kauranen, M. Boundary Element Method for Surface Nonlinear Optics of Nanoparticles. Opt. Express 2011, 19, 23386-23399.

(40) Butet, J.; Gallinet, B.; Thyagarajan, K.; Martin, O. J. F. SecondHarmonic Generation from Periodic Arrays of Arbitrary Shape Plasmonic Nanostructures: A Surface Integral Approach. J. Opt. Soc. Am. B 2013, 30, 2970-2979.

(41) Forestiere, C.; Capretti, A.; Miano, G. Surface Integral Method for Second Harmonic Generation in Metal Nanoparticles Including Both Local-Surface and Nonlocal-Bulk Sources. J. Opt. Soc. Am. B 2013, 30, 2355-2364.

(42) Thyagarajan, K.; Santschi, C.; Langlet, P.; Martin, O. J. F. Highly Improved Fabrication of $\mathrm{Ag}$ and $\mathrm{Al}$ Nanostructures for $\mathrm{UV}$ and Nonlinear Plasmonics. Adv. Opt. Mater. 2016, 4, 871-876.

(43) Kern, A. M.; Martin, O. J. F. Excitation and Reemission of Molecules Near Realistic Plasmonic Nanostructures. Nano Lett. 2011, 11, 482-487.

(44) Mühlig, S.; Menzel, C.; Rockstuhl, C.; Lederer, F. Multipole Analysis of Meta-Atoms. Metamaterials 2011, 5, 64-73.

(45) Nappa, J.; Revillod, G.; Russier-Antoine, I.; Benichou, E.; Jonin, C.; Brevet, P. F. Electric Dipole Origin of the Second Harmonic Generation of Small Metallic Particles. Phys. Rev. B: Condens. Matter Mater. Phys. 2005, 71, 165407.

(46) Russier-Antoine, I.; Benichou, E.; Bachelier, G.; Jonin, C.; Brevet, P.-F. Multipolar Contributions of the Second Harmonic Generation from Silver and Gold Nanoparticles. J. Phys. Chem. C 2007, 111, 9044.

(47) Mäkitalo, J.; Kauranen, M.; Suuriniemi, S. Modes and Resonances of Plasmonic Scatterers. Phys. Rev. B: Condens. Matter Mater. Phys. 2014, 89, 165429.

(48) Knight, M. W.; King, N. S.; Liu, L.; Everitt, H. O.; Nordlander, P.; Halas, N. J. Aluminum for Plasmonics. ACS Nano 2014, 8, 834840.

(49) Martin, J.; Plain, J. Fabrication of Aluminium Nanostructures for Plasmonics. J. Phys. D: Appl. Phys. 2015, 48, 184002.

(50) Martin, J.; Kociak, M.; Mahfoud, Z.; Proust, J.; Gérard, D.; Plain, J. High-Resolution Imaging and Spectroscopy of Multipolar Plasmonic Resonances in Aluminum Nanoantennas. Nano Lett. 2014, 14, 55175523.

(51) Cerjan, B.; Yang, X.; Nordlander, P.; Halas, N. J. Asymmetric Aluminum Antennas for Self-Calibrating Surface-Enhanced Infrared Absorption Spectroscopy. ACS Photonics 2016, 3, 354-360.

(52) Palik, E. D. Handbook of Optical Constants of Solids; Academic Press: New York, 1985.

(53) King, N. S.; Liu, L.; Yang, X.; Everitt, H. O.; Nordlander, P.; Halas, N. J. Fano Resonant Aluminum Nanoclusters for Plasmonic Colorimetric Sensing. ACS Nano 2015, 9, 10628-10636.

(54) McClain, M. J.; Schlather, A. E.; Ringe, E.; King, N. S.; Liu, L.; Manjavacas, A.; Knight, M. W.; Kumar, I.; Whitmire, K. H.; Everitt, H. O.; Nordlander, P.; Halas, N. J. Aluminum Nanocrystals. Nano Lett. 2015, 15, 2751-2755. 